\title{
Critically III Patients with Middle East Respiratory Syndrome Coronavirus Infection
}

\author{
H. M. Al-Dorzi, S. Alsolamy, and Y. M. Arabi
}

\section{Introduction}

The Middle East respiratory syndrome coronavirus (MERS-CoV) is an emerging virus that may lead to severe acute respiratory illness frequently associated with multiorgan failure and death. The objective of this chapter is to summarize the current state of knowledge regarding the pathogenesis, clinical manifestations, diagnosis, management and outcomes of MERS-CoV infection focusing on the critically ill.

\section{Epidemiology}

The virus was first isolated from a patient with fatal pneumonia and acute kidney injury in Jeddah, Saudi Arabia in June 2012 [1]. After its identification, the virus was linked to a healthcare-associated cluster of respiratory illnesses in Jordan dat-

\section{H. M. Al-Dorzi}

Intensive Care Department, King Saud bin Abdulaziz University for Health Sciences (KSAU-HS) Riyadh, Saudi Arabia

King Abdullah International Medical Research Center (KAIMRC)

Riyadh, Saudi Arabia

\section{S. Alsolamy}

Emergency Medicine and Intensive Care Departments, King Saud bin Abdulaziz University for Health Sciences (KSAU-HS)

Riyadh, Saudi Arabia

King Abdullah International Medical Research Center (KAIMRC)

Riyadh, Saudi Arabia

Y. M. Arabi $(\varangle)$

Intensive Care Department, King Saud bin Abdulaziz University for Health Sciences (KSAU-HS) Riyadh, Saudi Arabia

King Abdullah International Medical Research Center (KAIMRC)

Riyadh, Saudi Arabia

email: arabi@ngha.med.sa

J.-L. Vincent (ed.), Annual Update in Intensive Care and Emergency Medicine 2016,

DOI 10.1007/978-3-319-27349-5_4 
ing back to March 2012 [2]. The disease epidemiology has been characterized by sporadic cases throughout the year with surges of cases occurring because of hospital outbreaks. As of December 27, 2015, the World Health Organization (WHO) had reported 1,621 laboratory-confirmed cases, including at least $584(36 \%)$ related deaths [3]. Cases of MERS-CoV have occurred in 26 countries, with all cases linked to residence in or travel to the Arabian Peninsula.

The majority of cases have occurred in Saudi Arabia (80\%) and South Korea (12\%) [3]. In Saudi Arabia, multiple hospital outbreaks occurred in Alahsa (April-May 2013) [4], Jeddah (April and May 2014) [5], and Riyadh (AugustSeptember 2015) [3]. The outbreak in the Republic of Korea started in May 2015 and resulted from a case with travel history to the Middle East (Saudi Arabia, Qatar, United Arab Emirates and Bahrain). Subsequently, human-to-human transmission occurred to close contacts (family members, patients sharing a room or ward with infected patients and healthcare workers caring for infected patients) and led to 185 cases of MERS-CoV infection [3]. The last laboratory-confirmed case in Korea was on 4 July 2015 [3].

\section{Pathogenesis}

Coronaviruses are a family of enveloped, single-stranded RNA viruses [6]. They can infect animals and humans and have the propensity to cross species [6]. They cause a variety of illnesses that range from the common cold to severe respiratory illnesses [6]. The Severe Acute Respiratory Syndrome coronavirus (SARS-CoV) was the first coronavirus identified to cause widespread outbreaks of critical illness. It caused a worldwide outbreak during the winter of 2002-2003, eventually leading to 8096 confirmed cases, including 774 deaths [7]. The SARS epidemic was contained in 2003 [7]. SARS-CoV belongs to lineage B of the betacoronavirus [8], whereas MERS-CoV belongs to lineage $\mathrm{C}$ of the same genus. MERS$\mathrm{CoV}$ enters cells via a common receptor, the dipeptidyl peptidase-4 (DPP4), also known as CD26, and replicates in bronchial, bronchiolar and type I and II alveolar epithelial cells $[9,10]$. The virus has primarily been detected in respiratory secretions, with the highest viral loads in the lower respiratory tract [11]. In MERS$\mathrm{CoV}$ cases, one study showed that approximately $70 \%$ of patients had continued respiratory shedding for $>30$ days [12]. MERS-CoV infection often manifests with acute kidney injury (AKI), the pathogenesis of which remains unknown, although direct viral infection is a possibility since the DPP4 receptor is heavily expressed in human kidneys [13]. Viral shedding has also been detected in the urine and stool of infected patients [14].

There is accumulating evidence that camels are the primary source for animalto-human transmission of MERS-CoV. The virus has been isolated from dromedary camels [15]. The high viral loads in the upper respiratory tract of infected camels suggest that transmission to humans occurs through close camel contact [15]. Transmission through ingestion of camel products (meat and milk) is possible but has never been confirmed. In a cross-sectional serosurveillance study of 10,009 
individuals in Saudi Arabia, positive serology was documented in only $0.15 \%$ [16]. Seropositivity was more common in men than in women, in central than in coastal provinces and in shepherds and slaughterhouse workers than in others [16]. This may explain, at least in part, the sporadic community-acquired cases seen at low rates throughout the year.

Human-to-human transmission of MERS-CoV has been demonstrated among close household contacts and family members [17]. However, sustained community-based transmission has not been reported. Secondary transmission within healthcare settings has been described with already-hospitalized patients and healthcare workers acquiring the disease $[18,19]$. This has been attributed to overcrowding, movement of infected patients who did not yet have a diagnosis and breaches of infection prevention and control practices [3]. A study evaluated the stability of MERS-CoV under different environmental conditions and found that the virus was more stable at low temperature/low humidity than at higher temperature/ higher humidity conditions [20]. MERS-CoV could be recovered after $48 \mathrm{~h}$ at a $20^{\circ} \mathrm{C} / 40 \%$ relative humidity condition [20]. During MERS-CoV aerosolization, no decrease in stability was observed at the $20^{\circ} \mathrm{C} / 40 \%$ relative humidity condition [20]. The prolonged environmental presence suggests that MERS-CoV may be transmitted via contact or fomites [20], although this has not been confirmed.

\section{Clinical Manifestations}

The disease spectrum ranges from asymptomatic infection to rapidly progressive severe respiratory failure with multiorgan failure. Symptomatic cases manifest after an incubation period of 2-14 days [21]. Most severe cases have been reported in adults with chronic comorbidities, including diabetes mellitus, chronic cardiac disease, chronic lung disease, end-stage kidney disease, or immunosuppression [21, 22]. However, severe infection may also occur among younger patients, including healthcare workers [18]. Compared with SARS-CoV, MERS-CoV affects older patients, men more than women, and people with comorbid illnesses. The healthcareassociated MERS-CoV infections occurred within the first week of the index case illness, while for SARS they occurred mainly in the second week, likely due to the different timings of peak viral load [23]. The current literature suggests that children are rarely affected by MERS-CoV [24].

The most common clinical features in critically ill patients with MERS-CoV are fever (71\%), cough (68\%), dyspnea (66\%), and gastrointestinal symptoms (32\%) [21]. Common laboratory abnormalities include leukopenia, lymphocytopenia, and thrombocytopenia, elevated serum creatinine and lactate dehydrogenase, and altered liver enzymes [21]. Initial chest radiographs are abnormal in most symptomatic patients and findings range from minimal abnormalities to extensive bilateral infiltrates [21]. Figure 1 shows the chest radiographs of four patients with MERS-CoV pneumonia on the first day of intubation and demonstrates different patterns of lung infiltrates. Computed tomography (CT) findings have included bi- 
H. M. Al-Dorzi et al.

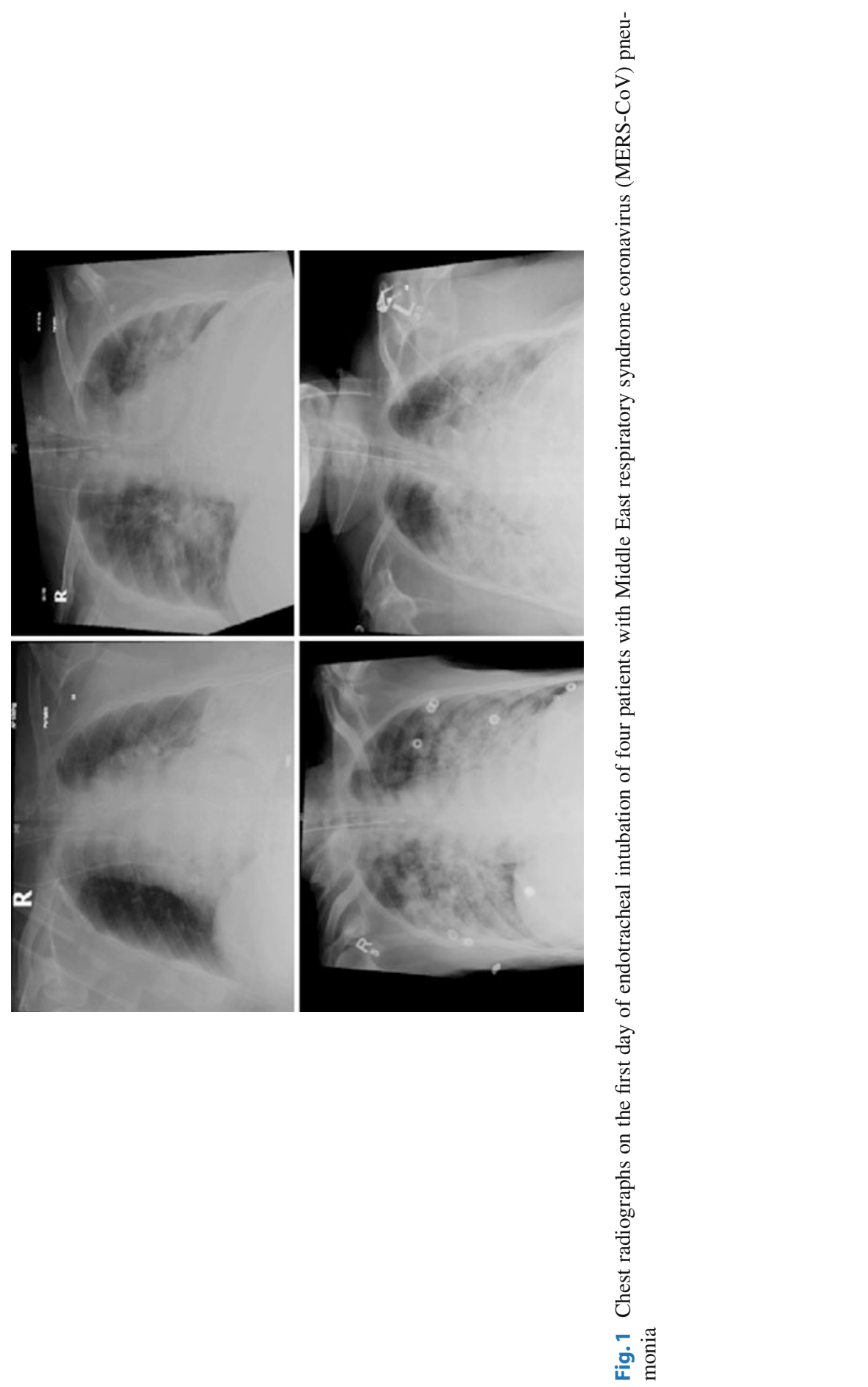


lateral airspace opacities predominantly in the subpleural and basilar lung regions with more extensive ground-glass opacities than consolidation [25].

Rapid progression to hypoxemic respiratory failure requiring intubation usually occurs within the first week after symptom onset [26]. In one observational study at a single center in Saudi Arabia (October 1, 2012 to May 31, 2014), severe infection requiring ICU admission occurred in 49 (70\%) of 70 cases with $46(66 \%)$ requiring invasive mechanical ventilation. In the univariate analysis, factors associated with severe infection requiring ICU admission were age $\geq 65$ years (odds ratio [OR] 9.47, 95\% confidence interval [CI] 2.45-36.56), male sex (OR 3.05, 95\% CI 1.05-8.84), higher age-adjusted Charlson comorbidity index (OR 1.35, 95\% CI 1.11-1.65), the presence of bilateral pulmonary infiltrates on chest radiograph (OR 4.89, 95\% CI 1.16-20.47), concomitant infections (OR 12.66, 95\% CI 2.65-60.46), and serum albumin <35 g/l at MERS-CoV diagnosis (OR 8.0, 95\% CI 1.97-32.46) [19]. Severe neurologic syndrome, including altered level of consciousness, ranging from confusion to coma, ataxia, and focal motor deficit, has been reported in three critically ill MERS-CoV patients, with brain magnetic resonance imaging (MRI) showing widespread, bilateral hyperintense lesions on T2-weighted images in the white matter and subcortical areas of different brain areas [27].

\section{Diagnosis}

The WHO has issued guidance for the definition of MERS-CoV cases, based on clinical criteria, exposure history and diagnostic findings as shown in Table 1 [3]. The clinical and radiological manifestations do not differentiate MERS-CoV infection from other causes of respiratory infection. Diagnosis is, therefore, based on molecular testing by real-time reverse-transcription polymerase chain reaction (rRT-PCR) for two sites in the virus genome: the upstream E protein (upE) for screening and the open reading frame (ORF) $1 \mathrm{a}$ or $1 \mathrm{~b}$ for confirmation [28]. Lower respiratory tract specimens have a higher sensitivity than upper respiratory tract specimens for detecting MERS-CoV [29]. The success of rRT-PCR testing depends on the experience and expertise of laboratory personnel, avoidance of contamination and the type and specimen condition. Gene sequencing targeting $R d R p$ (present in all corona viruses) and $N$ (specific to MERS-CoV) gene fragments can be used for confirmation [28]. MERS-CoV can be also diagnosed by seroconversion on two samples two weeks apart. Enzyme-linked immunosorbent assay can be used for screening and immunofluorescence assay (IFA) or neutralization for confirmation [28]. Several MERS-CoV-specific serologic assays have been developed but need validation [28]. The MERS-CoV virus can be cultured in commonly available cell lines [28]; however, this requires specialized biosafety level 3 laboratories. 
Table 1 Interim case definitions for Middle East respiratory syndrome coronavirus (MERS-CoV) infection (as of 14 July 2015) [3]

\begin{tabular}{|c|c|}
\hline \multirow[t]{3}{*}{ Probable case } & $\begin{array}{l}\text { An acute respiratory illness with fever and clinical, radiological, or } \\
\text { histopathological evidence of pulmonary involvement } \\
\text { AND } \\
\text { Direct epidemiologic link with a confirmed MERS-CoV case } \\
\text { AND } \\
\text { MERS-CoV testing is unavailable, negative on a single inadequate } \\
\text { specimen or inconclusive }\end{array}$ \\
\hline & $\begin{array}{l}\text { An acute respiratory illness with fever and clinical, radiological, or } \\
\text { histopathological evidence of pulmonary involvement } \\
\text { AND } \\
\text { The person lives in or has travelled to Middle Eastern countries or } \\
\text { countries where MERS-CoV is known to be circulating in dromedary } \\
\text { camels or where human MERS-CoV infections have recently occurred } \\
\text { AND } \\
\text { MERS-CoV testing is inconclusive }\end{array}$ \\
\hline & $\begin{array}{l}\text { An acute febrile respiratory illness of any severity } \\
\text { AND } \\
\text { Direct epidemiologic link with a confirmed MERS-CoV case } \\
\text { AND } \\
\text { MERS-CoV testing is inconclusive }\end{array}$ \\
\hline Confirmed case & $\begin{array}{l}\text { A person with laboratory-confirmed MERS-CoV infection irrespective } \\
\text { of clinical signs and symptoms }\end{array}$ \\
\hline
\end{tabular}

\section{Management}

At present, there is no proven specific therapy for MERS-CoV infection. Therefore, the mainstay of management of critically ill patients with MERS-CoV infection is supportive evidence-based care. Admission to the ICU is frequently required for close monitoring (e.g., patients with high oxygen requirements) or organ support. The WHO has issued interim guidance for the management of suspected and confirmed MERS-CoV infection [30].

\section{Infection Prevention and Control}

Because of the risk of transmission within the healthcare setting, appropriate patient isolation and strict implementation of infection prevention and control measures are crucial in the management of MERS-CoV cases. For suspected cases, the WHO recommends droplet and contact precautions [30]. When performing an aerosol generating procedure (e.g., aspiration or open suctioning of respiratory tract, intubation, bronchoscopy, cardiopulmonary resuscitation) airborne precautions should be additionally applied [30]. The Centers for Disease Control and Prevention (CDC) recommends that droplet precautions should be added to standard precautions when providing care to all patients with symptoms of acute respiratory infection and that a 
suspected or confirmed MERS-CoV case should be isolated in an airborne infection isolation room that is constructed and maintained according to current guidelines [31]. If such a room is not available, the patient should be transferred as soon as is feasible to a facility where one is available [31]. Until transfer, the patient should wear a facemask and should be isolated in a room with the door closed [31]. Healthcare workers should adhere to standard contact and airborne precautions. Hand hygiene should be performed appropriately and personal protective equipment, including respirators, should be applied correctly [31]. In addition, visitation should be restricted and controlled [31]. Hospitals should also develop protocols to be ready for a potential increase in the need to isolate patients with suspected or confirmed MERS-Cov infection.

\section{Management of Respiratory Failure}

Early supportive management includes supplemental oxygen for hypoxemia, respiratory distress and shock and early invasive mechanical ventilation for significant respiratory distress or persistent hypoxemia [30]. Patients with acute respiratory distress syndrome (ARDS) should receive evidence-based care that includes a lungprotective ventilation strategy [32]: targeting a tidal volume of $6 \mathrm{ml} / \mathrm{kg}$ of predicted body weight, a plateau airway pressure $\leq 30 \mathrm{cmH}_{2} \mathrm{O}$ and $\mathrm{SpO}_{2} 88-93 \%$ or $\mathrm{PaO}_{2}$ $55-80 \mathrm{mmHg}$. Moderate to severe ARDS cases $\left(\mathrm{PaO}_{2}: \mathrm{FiO}_{2}<150 \mathrm{mmHg}\right)$ are candidates for early prone positioning [33] and early neuromuscular blockade for $48 \mathrm{~h}$ [34]. Proning is recommended within $36 \mathrm{~h}$ of ARDS onset for at least $16 \mathrm{~h}$, since this approach was associated with reduced mortality compared with managing patients in the supine position [33]. A 48-h infusion of intravenous cisatracurium within $48 \mathrm{~h}$ of ARDS onset has also been associated with reduced mortality compared with placebo [34]. Extracorporeal membrane oxygenation (ECMO) is an option for patients with refractory hypoxemia [35, 36], and has been successfully used in young patients with severe H1N1 influenza with refractory hypoxemia [36] as well as in MERS-CoV patients [11]. Systematic corticosteroids should generally be avoided unless indicated for other established reasons [30]. The effect of corticosteroids on the outcome of MERS-CoV patients is unknown; however, corticosteroid use has been associated with increased morbidity in patients with SARS$\mathrm{CoV}$ and severe influenza $[37,38]$.

High-flow oxygen, non-invasive ventilation and high-frequency oscillatory ventilation should probably be avoided or used with caution in MERS patients because of the potential to generate aerosols and lack of effectiveness in patients with ARDS [39]. A systematic review found that the following procedures were associated with an increased risk of acute respiratory infection transmission to healthcare workers through aerosol-generation: endotracheal intubation, non-invasive ventilation, tracheotomy, and manual ventilation [39]. Strict infection prevention practices should, therefore, be employed when these interventions are performed. 


\section{Management of Acute Kidney Injury}

Conservative fluid management is advocated especially in the presence of hypoxemia and the absence of shock. When renal replacement therapy (RRT) should be started in patients with AKI is controversial. However, the presence of fluid overload at the start of RRT has been associated with a worse outcome [40]. Starting ultrafiltration at lower degrees of fluid overload and early targeting of a negative balance may be beneficial $[41,42]$.

\section{Feeding}

Early trophic feeding for 7 days in ARDS patients [43] and permissive underfeeding for up to 14 days in mechanically ventilated patients [44] have been shown to be equivalent to target feeding for most outcomes including survival. Underfeeding may be preferred for patients in the prone position.

\section{Specific Therapies}

To date, there is no effective specific treatment for MERS-CoV infection. Data on ribavirin, interferon, and convalescent plasma are limited [21]. A study in rhesus macaques found that a combination of interferon- $\alpha 2 b$ and ribavirin was associated with a reduction in MERS-CoV replication and improved outcome [45]. Clinical studies on combined ribavirin and interferon showed inconsistent results [46]. Convalescent sera from patients who have recovered may be useful. A post-hoc metaanalysis of 32 studies on SARS-CoV and severe influenza found reduced mortality after antibody treatment compared with placebo or no treatment (pooled OR $0.25,95 \%$ CI $0.14-0.45$ ), but the included studies were of low or very low quality [47]. Three studies from separate laboratories have reported the development of fully human neutralizing monoclonal antibodies against MERS-CoV but no clinical data are currently available [48]. Repurposed commonly-used drugs, such as statins, mycophenolic acid, and anti-tumor necrosis factor agents, which have potential anti-inflammatory or antiviral effects against MERS-CoV and at the same time an excellent safety record, may be treatment options. However, these drugs are not recommended for clinical use outside clinical trials [49]. There are no currently licensed MERS-CoV vaccines; however, work is ongoing to develop effective vaccines [50].

\section{Outcomes}

The overall case fatality rate of MERS-CoV infection is 35\% [3]. However, the mortality rate of patients with severe illness requiring critical care is higher $(58-84 \%)[19,22,26]$. Requirement for ICU admission for hospitalized patients 
is frequent $(45-70 \%)[19,22]$. In an observational study, 41 out of $49(84 \%)$ cases requiring ICU admission died, with 33 (78.6\%) having a progressive disease course until death [19]. In a multivariable regression analysis, only the presence of a concomitant infection (OR 14.13, 95\% CI 1.58-126.09) and a low serum albumin (OR 6.31, 95\% CI 1.24-31.90) were associated with the need for ICU admission [19]. The multivariable analysis showed age $\geq 65$ years to be the only independent risk factor for increased mortality (OR 4.39, 95\% CI 2.13-9.05) [19]. AKI $(41 \%)$, hepatic dysfunction (31\%) and cardiac arrhythmias (16\%), such as tachyarrhythmias and severe bradycardia requiring temporary pacemaker insertion, were common complications [19]. Another observational study of 12 critically ill cases found that barotrauma occurred in $17 \%$, vasopressors were needed in $92 \%$ and RRT was performed in 58\% [26]. The median time from onset of symptoms to intubation was 4.5 days (range 0-33 days). The median ICU and hospital lengths of stay were 30 (7-104) days and 41 (8-96) days, respectively, and $25 \%$ of patients had a tracheostomy [26]. The 28-day and 90-day mortality rates were 42 and 58\%, respectively [26].

It should be noted that the mortality of MERS-CoV cases during the Korean outbreak (May-July 2015) was only 19\% compared with 42\% in Saudi Arabia (as at Sept 11, 2015) [3]. However, the composition of the two cohorts was different in the relative percentages of asymptomatic and mildly symptomatic cases. The Korean healthcare authorities applied widespread screening and intensified public health measures, such as contact tracing, quarantine and isolation of suspected cases and all contacts for at least 14 days [3]. The identification of asymptomatic or mildly symptomatic patients and differences in patient characteristics, such as comorbid conditions, may have led to an overall lower mortality rate in Korea.

\section{Conclusions and Future Directions}

More than three years after its identification, MERS-CoV remains a major global threat. Infection with MERS-CoV is frequently severe and associated with significant morbidity and mortality. Although our understanding of this virus and its disease continues to evolve, large knowledge gaps remain. The Arabian Peninsula remains the most affected region, but there is concern about the spread of this virus to other countries and globally. Until zoonotic transfer of the virus from animals to humans is stopped, the risk of additional domestic and perhaps international outbreaks persists. Moreover, there is an urgent need for larger epidemiologic and outcome studies and randomized controlled trials examining different therapeutic interventions. Collaboration between international health authorities and research centers is necessary and is crucial to achieve these goals. 


\section{References}

1. Zaki AM, van Boheemen S, Bestebroer TM, Osterhaus AD, Fouchier RA (2012) Isolation of a novel coronavirus from a man with pneumonia in Saudi Arabia. N Engl J Med 367:1814-1820

2. Hijawi B, Abdallat M, Sayaydeh A et al (2013) Novel coronavirus infections in Jordan, April 2012: epidemiological findings from a retrospective investigation. East Mediterr Health J 19(Suppl 1):S12-S18

3. World Health Organization (2015) Middle East respiratory syndrome coronavirus (MERSCoV). http://www.who.int/csr/disease/coronavirus_infections/case_definition/en/. Accessed December 2015

4. Assiri A, McGeer A, Perl TM et al (2013) Hospital outbreak of Middle East respiratory syndrome coronavirus. N Engl J Med 369:407-416

5. Oboho IK, Tomczyk SM, Al-Asmari AM et al (2015) 2014 MERS-CoV outbreak in Jeddah - a link to health care facilities. N Engl J Med 372:846-854

6. Abdel-Moneim AS (2014) Middle East respiratory syndrome coronavirus (MERS-CoV): evidence and speculations. Arch Virol 159:1575-1584

7. World Health Organization (2003) Summary of probable SARS cases with onset of illness from 1 November 2002 to 31 July 2003. http://www.who.int/csr/sars/country/table2004_04_ 21/en/. Accessed December 2015

8. van Boheemen S, de Graaf M, Lauber C et al (2012) Genomic characterization of a newly discovered coronavirus associated with acute respiratory distress syndrome in humans. MBio 3:1-9

9. Hocke AC, Becher A, Knepper J et al (2013) Emerging human middle East respiratory syndrome coronavirus causes widespread infection and alveolar damage in human lungs. Am J Respir Crit Care Med 188:882-886

10. Chan RW, Chan MC, Agnihothram S et al (2013) Tropism of and innate immune responses to the novel human betacoronavirus lineage $\mathrm{C}$ virus in human ex vivo respiratory organ cultures. J Virol 87:6604-6614

11. Guery B, Poissy J, el Mansouf L et al (2013) Clinical features and viral diagnosis of two cases of infection with Middle East Respiratory Syndrome coronavirus: a report of nosocomial transmission. Lancet 381:2265-2272

12. Memish ZA, Assiri AM, Al-Tawfiq JA (2014) Middle East respiratory syndrome coronavirus (MERS-CoV) viral shedding in the respiratory tract: an observational analysis with infection control implications. Int J Infect Dis 29:307-308

13. Eckerle I, Muller MA, Kallies S, Gotthardt DN, Drosten C (2013) In-vitro renal epithelial cell infection reveals a viral kidney tropism as a potential mechanism for acute renal failure during Middle East Respiratory Syndrome (MERS) Coronavirus infection. Virol J 10:359

14. Drosten C, Seilmaier M, Corman VM et al (2013) Clinical features and virological analysis of a case of Middle East respiratory syndrome coronavirus infection. Lancet Infect Dis 13:745751

15. Azhar EI, El-Kafrawy SA, Farraj SA et al (2014) Evidence for camel-to-human transmission of MERS coronavirus. N Engl J Med 370:2499-2505

16. Muller MA, Meyer B, Corman VM et al (2015) Presence of Middle East respiratory syndrome coronavirus antibodies in Saudi Arabia: a nationwide, cross-sectional, serological study. Lancet Infect Dis 15:559-564

17. Memish ZA, Zumla AI, Al-Hakeem RF, Al-Rabeeah AA, Stephens GM (2013) Family cluster of Middle East respiratory syndrome coronavirus infections. N Engl J Med 368:2487-2494

18. Memish ZA, Zumla AI, Assiri A (2013) Middle East respiratory syndrome coronavirus infections in health care workers. N Engl J Med 369:884-886

19. Saad M, Omrani AS, Baig K et al (2014) Clinical aspects and outcomes of 70 patients with Middle East respiratory syndrome coronavirus infection: a single-center experience in Saudi Arabia. Int J Infect Dis 29:301-306 
20. van Doremalen N, Bushmaker T, Munster VJ (2013) Stability of Middle East respiratory syndrome coronavirus (MERS-CoV) under different environmental conditions. Euro Surveill $18: 1-4$

21. Alsolamy S (2015) Middle East respiratory syndrome: knowledge to date. Crit Care Med 43:1283-1290

22. Al-Tawfiq JA, Hinedi K, Ghandour J et al (2014) Middle East respiratory syndrome coronavirus: a case-control study of hospitalized patients. Clin Infect Dis 59:160-165

23. Hui DS, Memish ZA, Zumla A (2014) Severe acute respiratory syndrome vs. the Middle East respiratory syndrome. Curr Opin Pulm Med 20:233-241

24. Memish ZA, Al-Tawfiq JA, Assiri A et al (2014) Middle East respiratory syndrome coronavirus disease in children. Pediatr Infect Dis J 33:904-906

25. Ajlan AM, Ahyad RA, Jamjoom LG, Alharthy A, Madani TA (2014) Middle East respiratory syndrome coronavirus (MERS-CoV) infection: chest CT findings. AJR Am J Roentgenol 203:782-787

26. Arabi YM, Arifi AA, Balkhy HH et al (2014) Clinical course and outcomes of critically ill patients with Middle East respiratory syndrome coronavirus infection. Ann Intern Med 160:389397

27. Arabi YM, Harthi A, Hussein J et al (2015) Severe neurologic syndrome associated with Middle East respiratory syndrome corona virus (MERS-CoV). Infection 43:495-501

28. Corman VM, Muller MA, Costabel U et al (2012) Assays for laboratory confirmation of novel human coronavirus (hCoV-EMC) infections. Euro Surveill 17:1-9

29. Lee JH, Lee CS, Lee HB (2015) An appropriate lower respiratory tract specimen is essential for diagnosis of Middle East Respiratory Syndrome (MERS). J Korean Med Sci 30:1207-1208

30. World Health Organization (2015) Clinical management of severe acute respiratory infection when Middle East respiratory syndrome coronavirus (MERS-CoV) infection is suspected Interim guidance. www.who.int/csr/disease/coronavirus_infections/case-management-ipc/en/. Accessed December 2015

31. Centers for Disease Control and Prevention (2014) Interim Infection Prevention and Control Recommendations for Hospitalized Patients with Middle East Respiratory Syndrome Coronavirus (MERS-CoV). http://www.cdc.gov/coronavirus/mers/infection-prevention-control. html. Accessed December 2015

32. Petrucci N, De Feo C (2013) Lung protective ventilation strategy for the acute respiratory distress syndrome. Cochrane Database Syst Rev 2:CD003844

33. Guerin C, Reignier J, Richard JC et al (2013) Prone positioning in severe acute respiratory distress syndrome. N Engl J Med 368:2159-2168

34. Papazian L, Forel JM, Gacouin A et al (2010) Neuromuscular blockers in early acute respiratory distress syndrome. N Engl J Med 363:1107-1116

35. Peek GJ, Mugford M, Tiruvoipati R et al (2009) Efficacy and economic assessment of conventional ventilatory support versus extracorporeal membrane oxygenation for severe adult respiratory failure (CESAR): a multicentre randomised controlled trial. Lancet 374:1351-1363

36. Davies A, Jones D, Bailey M et al (2009) Extracorporeal membrane oxygenation for 2009 Influenza A (H1N1) acute respiratory distress syndrome. JAMA 302:1888-1895

37. Martin-Loeches I, Lisboa T, Rhodes A et al (2011) Use of early corticosteroid therapy on ICU admission in patients affected by severe pandemic (H1N1)v influenza A infection. Intensive Care Med 37:272-283

38. Stockman LJ, Bellamy R, Garner P (2006) SARS: systematic review of treatment effects. PLoS Med 3:1525-1531

39. Tran K, Cimon K, Severn M, Pessoa-Silva CL, Conly J (2012) Aerosol generating procedures and risk of transmission of acute respiratory infections to healthcare workers: a systematic review. PLoS One 7:e35797 
40. Bellomo R, Cass A, Cole L et al (2012) An observational study fluid balance and patient outcomes in the Randomized Evaluation of Normal vs. Augmented Level of Replacement Therapy trial. Crit Care Med 40:1753-1760

41. Ronco C, Ricci Z, De Backer D et al (2015) Renal replacement therapy in acute kidney injury: controversy and consensus. Crit Care 19:146

42. Han F, Sun R, Ni Y et al (2015) Early initiation of continuous renal replacement therapy improves clinical outcomes in patients with acute respiratory distress syndrome. Am J Med Sci 349:199-205

43. Rice TW, Wheeler AP, Thompson BT et al (2012) Initial trophic vs full enteral feeding in patients with acute lung injury: the EDEN randomized trial. JAMA 307:795-803

44. Arabi YM, Aldawood AS, Haddad SH et al (2015) Permissive underfeeding or standard enteral feeding in critically ill adults. N Engl J Med 372:2398-2408

45. Falzarano D, de Wit E, Rasmussen AL et al (2013) Treatment with interferon-alpha2b and ribavirin improves outcome in MERS-CoV-infected rhesus macaques. Nat Med 19:1313-1317

46. Al-Tawfiq JA, Momattin H, Dib J, Memish ZA (2014) Ribavirin and interferon therapy in patients infected with the Middle East respiratory syndrome coronavirus: an observational study. Int J Infect Dis 20:42-46

47. Mair-Jenkins J, Saavedra-Campos M, Baillie JK et al (2015) The effectiveness of convalescent plasma and hyperimmune immunoglobulin for the treatment of severe acute respiratory infections of viral etiology: a systematic review and exploratory meta-analysis. J Infect Dis 211:80-90

48. Ying T, Li H, Lu L, Dimitrov DS, Jiang S (2015) Development of human neutralizing monoclonal antibodies for prevention and therapy of MERS-CoV infections. Microbes Infect $17: 142-148$

49. Zumla A, Azhar EI, Arabi Y et al (2015) Host-directed therapies for improving poor treatment outcomes associated with the middle east respiratory syndrome coronavirus infections. Int $\mathbf{J}$ Infect Dis 40:71-74

50. Zhang N, Jiang S, Du L (2014) Current advancements and potential strategies in the development of MERS-CoV vaccines. Expert Rev Vaccines 13:761-774 